\title{
Planetesimal formation starts at the snow line ${ }^{\star}$
}

\author{
J. Drążkowska ${ }^{1}$ and Y. Alibert ${ }^{2}$ \\ 1 Institute for Computational Science, University of Zurich, Winterthurerstrasse 190, 8057 Zurich, Switzerland \\ e-mail: joannad@physik.uzh.ch \\ 2 Physikalisches Institut \& Center for Space and Habitability, University of Bern, Sidlerstrasse 5, 3012 Bern, Switzerland
}

Received 3 July 2017 / Accepted 28 September 2017

\begin{abstract}
Context. The formation stage of planetesimals represents a major gap in our understanding of the planet formation process. Late-stage planet accretion models typically make arbitrary assumptions about planetesimal and pebble distribution, while dust evolution models predict that planetesimal formation is only possible at some orbital distances.

Aims. We wish to test the importance of the water snow line in triggering the formation of the first planetesimals during the gas-rich phase of a protoplanetary disk, when cores of giant planets have to form.

Methods. We connected prescriptions for gas disk evolution, dust growth and fragmentation, water ice evaporation and recondensation, the transport of both solids and water vapor, and planetesimal formation via streaming instability into a single one-dimensional model for protoplanetary disk evolution.

Results. We find that processes taking place around the snow line facilitate planetesimal formation in two ways. First, because the sticking properties between wet and dry aggregates change, a "traffic jam" inside of the snow line slows the fall of solids onto the star. Second, ice evaporation and outward diffusion of water followed by its recondensation increases the abundance of icy pebbles that trigger planetesimal formation via streaming instability just outside of the snow line.

Conclusions. Planetesimal formation is hindered by growth barriers and radial drift and thus requires particular conditions to take place. The snow line is a favorable location where planetesimal formation is possible for a wide range of conditions, but not in every protoplanetary disk model, however. This process is particularly promoted in large cool disks with low intrinsic turbulence and an increased initial dust-to-gas ratio.
\end{abstract}

Key words. accretion, accretion disks - circumstellar matter - protoplanetary disks - planets and satellites: formation methods: numerical

\section{Introduction}

Our understanding of planet formation is severely limited by the fact that we cannot explain the connection between its early and late stages. As a consequence, models of late-stage planet accretion, when a planetary embryo grows into its final size and structure, typically use the same input for the radial distribution of gas and solids as early-stage models of dust growth and planetesimal formation. However, the latter models show that growing large bodies is not easy because of the growth barriers: the dust growth is inhibited at centimeter sizes, and some particular conditions are needed for the formation of larger gravitationally bound planetesimals and planetary embryos.

Probably the currently most widely accepted planetesimal formation scenario is the streaming instability (Youdin \& Goodman 2005; Johansen et al. 2007). For sufficiently large pebbles and an increased metallicity (Bai \& Stone 2010; Dra̧żkowska \& Dullemond 2014; Carrera et al. 2015), streaming instability leads to the formation of dense filaments that become gravitationally unstable and collapse to form planetesimals. This scenario allows us to bypass the growth barriers and form gravitationally bound object directly from pebbles.

The streaming instability is typically simulated in local boxes because of the high computational cost of hydrodynamical

\footnotetext{
$\star$ The movie attached to Fig. 3 is only available at http://www . aanda.org
}

simulations (Johansen et al. 2011; Kowalik et al. 2013; Simon et al. 2016). Thus, the initial conditions are already set up in a way for a streaming instability to occur. However, these conditions are not necessarily met in a realistic disk that starts its evolution with a dust-to-gas ratio on the order of $1 \%$, which is depleted because solids are removed by the radial drift (Birnstiel et al. 2010; Hughes \& Armitage 2012; Krijt et al. 2016b). Pebble pile-ups may be necessary to allow for planet formation in the gas-rich phase of a protoplanetary disk (Drążkowska et al. 2016; Gonzalez et al. 2017), while disk dispersal via photoevaporation may allow for late planetesimal formation (Carrera et al. 2017). Both processes may be needed to explain the existence of different planet types and debris belts, for instance, in the solar system. In this paper, we focus on the former mechanism, with the aim of triggering planetesimal formation early in the evolution of gas disk in order to allow sufficient time for the formation of gas-rich planets.

The great dichotomy of the solar system used to be commonly attributed to the jump change of condition around the snow line (Stevenson \& Lunine 1988; Wuchterl et al. 2000; Morbidelli et al. 2015), with water ice greatly enhancing the abundance of solids outside of this point. A rapid change of conditions like this may contribute to a pressure bump buildup that would halt the radial drift of solids and thus facilitate planet formation (Kretke \& Lin 2007; Brauer et al. 2008; Drążkowska et al. 2013). It was also proposed that the icy dust 
aggregates can pile up (Cuzzi \& Zahnle 2004) or even significantly grow through water vapor recondensation at the snow line (Ros \& Johansen 2013; Wang 2015). Laboratory and numerical experiments that studied collisional properties of dust aggregates concluded that the icy aggregates are significantly more sticky than silicate grains (Wada et al. 2011; Gundlach et al. 2011) and can thus grow to larger sizes before they fragment, or even grow directly to planetesimal sizes if they are sufficiently porous (Okuzumi et al. 2012; Kataoka et al. 2013).

In this paper, we analyze how the snow line could trigger formation of the first gravitationally bound planetesimals at the very beginning of planet formation. We start our simulations with a smooth protoplanetary disk and let it evolve, taking into account dust growth to pebbles, their drift and fragmentation, and ice evaporation and recondensation. In order to demonstrate the universality of our findings, we test our scenario in three different protoplanetary disk models. We conclude that the water component has an immense effect on the growth and redistribution of solids and leads to a pile-up of icy pebbles and planetesimal formation via streaming instability just outside of the snow line. Significant contribution to this pile-up comes from the change of sticking properties between icy and dry aggregates, an effect that was previously included in some of the models (Birnstiel et al. 2010; Banzatti et al. 2015; Estrada et al. 2016; Cridland et al. 2017), but was not discussed directly in the context of planetesimal formation.

This paper is organized as follows. We describe our numerical modeling approach and typical initial conditions in Sect. 2 and present typical results and their dependence on input parameters in Sect. 3. We discuss the differences between our work and other published results as well as the implications of our findings in Sect. 4, and we finally summarize our work in Sect. 5.

\section{Model}

We implemented a one-dimensional protoplanetary disk model in which we follow the radial distribution of solids and formation of planetesimals over one million years. We focused on the gas-rich phase of protoplanetary disk, before photoevaporation is efficient, therefore we either included only viscous evolution or implemented a static gas disk. The initial dust grains size is $1 \mu \mathrm{m}$ at every orbital distance. We assumed that the dust may be composed of water ice and silicates, and that the initial ice mass fraction outside of the snow line is $50 \%$. At the beginning of each simulation, the ice and water vapor were distributed across the disk such that the vertically integrated water-to-gas ratio was uniform and equal to 0.5 of the total metallicity. Solid ice was only present outside of the snow line, and water vapor was present inside of the snow line. The refractory dust component was present both outside and inside of the snow line. We followed dust growth to pebbles, fragmentation, and radial drift, and ice evaporation and recondensation of water vapor.

The evolution of solids and water vapor is governed by their interactions with the sub-Keplerian turbulent gas. We treated the gas disk as a background for the solids evolution and plugged in different gas disk models, as described in the following section.

\subsection{Gas disk models}

To test versatility of the planetesimal formation scenario, we used three different protoplanetary disk models. All of them had a total gas mass of $0.1 M_{\odot}$ within $100 \mathrm{AU}$ distance to the central star of $M_{\star}=1 M_{\odot}$. Their other properties are described in
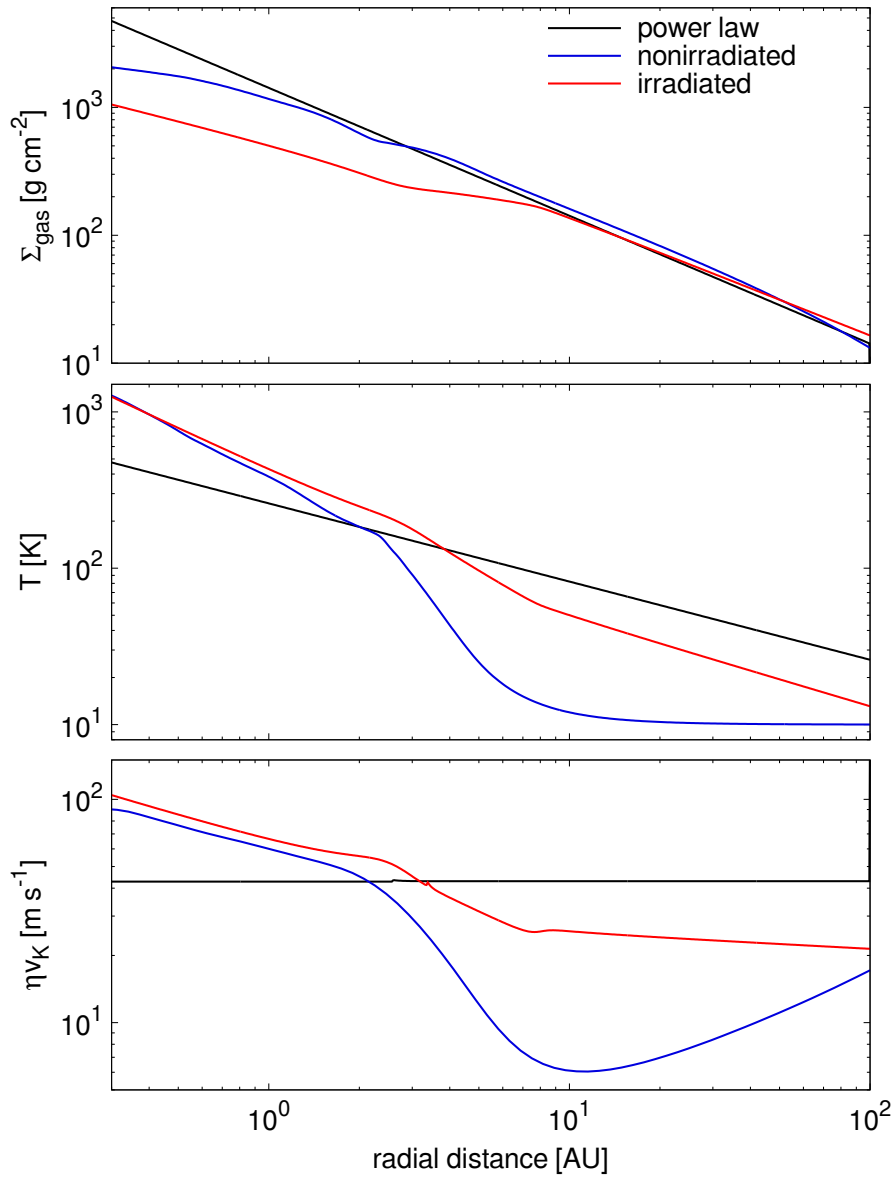

Fig. 1. Comparison of the initial conditions for our protoplanetary disk models: the power-law disk (black), the non-irradiated disk (blue), and the irradiated disk (red). The panels show, from top to bottom, the gas surface density $\Sigma_{\text {gas }}$, the temperature $T$, and the difference between gas and Keplerian rotation, which is equal to the maximum drift speed of dust pebbles $\eta v_{\mathrm{K}}$. Each of the disks has a total mass of $0.1 M_{\odot}$.

subsequent paragraphs. A comparison of the basic properties of these disks such as the surface density, temperature, and deviation from the Keplerian rotation is displayed in Fig. 1.

\subsubsection{Power-law disk}

Very simple protoplanetary disk models are commonly used in planet formation research. Here, we adopted one of them, with the gas surface density profile set as the straightforward function of the distance to the central star $r$

$\Sigma_{\mathrm{g}}=1400 \cdot\left(\frac{r}{\mathrm{AU}}\right)^{-1}$

and the temperature profile was fixed to

$T=150 \cdot\left(\frac{r}{3 \mathrm{AU}}\right)^{-1 / 2}$

For simplicity, we keep this disk static because the model is already basic, but we included effects of gas accretion velocity $v_{\mathrm{g}}$ when we calculated the radial drift of solids. This velocity is estimated as

$v_{\mathrm{g}}=\frac{3}{2} \frac{D_{\mathrm{g}}}{r}$ 
where turbulent gas diffusivity is equal to gas viscosity

$D_{\mathrm{g}}=v=\alpha_{\mathrm{v}} \frac{c_{\mathrm{s}}^{2}}{\Omega_{\mathrm{K}}}$,

which is calculated based on the standard $\alpha$-accretion model (Shakura \& Sunyaev 1973) with $\alpha_{\mathrm{v}}$ being the dimensionless parameter describing the efficiency of angular momentum transfer. We typically used $\alpha_{\mathrm{v}}=10^{-3}$ in this paper. The $\Omega_{\mathrm{K}}$ is the Keplerian orbital frequency, and the gas sound speed $c_{\mathrm{S}}$ is calculated as

$c_{\mathrm{s}}=\sqrt{\frac{k_{\mathrm{B}} T}{\mu}}$,

where $k_{\mathrm{B}}$ is the Boltzmann constant and $\mu$ is the mean molecular weight of gas. When calculating $\mu$, we included the contribution of water vapor:

$\mu=\left(\Sigma_{\mathrm{g}}+\Sigma_{\mathrm{H}_{2} \mathrm{O}}\right) \cdot\left(\frac{\Sigma_{\mathrm{g}}}{\mu_{\mathrm{g}}}+\frac{\Sigma_{\mathrm{H}_{2} \mathrm{O}}}{\mu_{\mathrm{H}_{2} \mathrm{O}}}\right)^{-1}$,

which increases the molecular weight and thus decreases the sound speed in the inner part of the protoplanetary disk. We took $\mu_{\mathrm{g}}=2.34 m_{\mathrm{p}}$ and $\mu_{\mathrm{H}_{2} \mathrm{O}}=18 m_{\mathrm{p}}$, with $m_{\mathrm{p}}$ denoting the proton mass. The effect of variable $\mu$ is visible in the bottom panel of Fig. 1 as a small jump of the maximum drift speed (equal to the difference between the gas and Keplerian rotation) around the snow line location. The maximum radial drift speed is roughly constant in the power-law disk model, with $\eta v_{\mathrm{K}} \approx 40 \mathrm{~m} \mathrm{~s}^{-1}$.

\subsubsection{Non-irradiated disk}

We compared the simple static power-law disk model to the more complex viscously evolving circumstellar disk model described in Alibert et al. (2005, 2013), which was also used in our previous work presented in Dra̧żkowska et al. (2016). In this model, the vertical structure of the disk is first computed for every distance to the star by solving the hydrostatic equation, the energy conservation equation, and the radiative diffusion equation (energy is assumed to be transported by radiation). The solution of the vertical structure equations gives the thermodynamical quantities (temperature, pressure, density, but also the disk scale height) as a function of the gas surface density. The same calculation also gives the vertically averaged viscosity, which is again computed in the framework of the $\alpha$ formalism. We finally solve the diffusion equation, the viscosity being the one derived from the vertical structure calculation, in order to compute the time evolution of the gas surface density.

The middle panel of Fig. 1 shows that this disk is very cold in its outer part, as the temperature drops down to $10 \mathrm{~K}$ outside of $10 \mathrm{AU}$. Inside of $10 \mathrm{AU}$, the temperature profile is steeper than the one implemented in the power-law disk and reaches over $1000 \mathrm{~K}$ at the inner edge of the disk. This steeper temperature profile leads to a flatter surface density in the inner part of the disk, while it is very similar to the power-law disk in the outer part. In the inner part of the disk, where the temperature profile is steep, the maximum radial drift speed is higher than for the power-law disk, but it drops in the outer disk, forming a wide minimum around $10 \mathrm{AU}$ (see the bottom panel of Fig. 1).

\subsubsection{Irradiated disk}

To test the effect of stellar irradiation, we implemented the simple analytical model proposed by Bitsch et al. (2015, B15 in the following), which was designed to fit $2 \mathrm{D}$ radiative hydrodynamic simulations of protoplanetary disks. In this model the disk evolves with time, and the accretion rate $\dot{M}$ decreases as follows (Hartmann et al. 1998)

$\log \left(\frac{\dot{M}}{M_{\odot} / \mathrm{yr}}\right)=-8.00-1.40 \cdot \log \left(\frac{t+10^{5} \mathrm{yr}}{10^{6} \mathrm{yr}}\right)$,

where $t$ is the evolution time and $\dot{M}$ is related to the viscosity and the gas surface density $\Sigma_{\mathrm{g}}$ via

$\dot{M}=3 \pi v \Sigma_{\mathrm{g}}=3 \pi \alpha_{\mathrm{v}} H_{\mathrm{g}}^{2} \Omega_{\mathrm{K}} \Sigma_{\mathrm{g}}$,

where $H_{\mathrm{g}}$ the scale height of the disk, and $\Omega_{\mathrm{K}}$ the Keplerian frequency. In this disk model, we used $\alpha_{\mathrm{v}}=0.0054$ following B15. We note that $\alpha_{\mathrm{v}}$ is only used in B15 as a heating parameter and not to evolve the disk viscously, as it is in the case of the non-irradiated disk model. For a given $\dot{M}$, all quantities of the disk can be derived, except for the temperature profile. In order to compute the latter, we used the formulas presented in Appendix A of B15.

After the temperature is determined, it can be linked to the disk aspect ratio via

$T=\left(\frac{H_{\mathrm{g}}}{r}\right)^{2} \frac{G M_{\star}}{r} \frac{\mu}{\mathcal{R}}$,

where $G$ is the gravitational constant, $r$ the location in the disk, $\mathcal{R}$ is the gas constant, and $\mu$ is the mean molecular weight.

Figure 1 shows that the temperature in this disk is significantly higher than in the non-irradiated disk, particularly in its outer part, therefore the surface density flattening is also more pronounced. In addition, the maximum drift speed is higher than in the non-irradiated disk, but still lower than in the power-law disk in the outer parts.

\subsection{Evolution of solids}

We followed the evolution of the solids surface density $\Sigma_{d}$ by solving the advection-diffusion equation

$\frac{\partial \Sigma_{\mathrm{d}}}{\partial t}+\frac{1}{r} \frac{\partial}{\partial r}\left[r\left(\Sigma_{\mathrm{d}} \bar{v}-D_{\mathrm{g}} \Sigma_{\mathrm{g}} \frac{\partial}{\partial r}\left(\frac{\Sigma_{\mathrm{d}}}{\Sigma_{\mathrm{g}}}\right)\right)\right]=0$,

where $\bar{v}$ is the mass-weighted average radial velocity of solids, which encompasses information about their size.

The dust aggregates sizes were modeled using the method based on the two-population algorithm proposed by Birnstiel et al. (2012). The logic behind this algorithm is to reduce computational intensity by not solving for the dust coagulation directly, but rather predict its outcome based on more complete models. The dust size distribution is therefore set in each radial grid cell depending on the dominating process: coagulation-fragmentation equilibrium or radial drift. We do not describe further details of this model as they are thoroughly outlined in the original work as well as in our previous paper (Dra̧żkowska et al. 2016). Importantly, the outcome of this algorithm is the size, or Stokes number, distribution at every radial distance. The Stokes number informs us about the interaction between the solids grain and gas, and is connected to the grain size a:

$\mathrm{St}=\frac{\pi}{2} \frac{a \rho_{\bullet}}{\Sigma_{\mathrm{g}}}$,

where $\rho_{\bullet}$ is the aggregate internal density. Equation (11) is derived under the assumption that the solids are in the Epstein drag 
regime, which means that their sizes do not exceed the mean free path in the gas, which is true for all our models. The internal density of aggregates is calculated based on their composition:

$\rho_{\bullet}=\left(m_{\text {sil }}+m_{\text {ice }}\right) \cdot\left(\frac{m_{\text {sil }}}{\rho_{\bullet, \text { sil }}}+\frac{m_{\text {ice }}}{\rho_{\bullet \text {,ice }}}\right)^{-1}$,

where $m_{\text {sil }}$ and $m_{\text {ice }}$ are mass of silicate and mass of ice contained within the aggregate. A pure water ice aggregate would have $\rho_{\bullet \text {,ice }}=1 \mathrm{~g} \mathrm{~cm}^{-3}$ and a silicate aggregate $\rho_{\bullet, \text { sil }}=3 \mathrm{~g} \mathrm{~cm}^{-3}$.

When calculating the advection speed of solids $\bar{v}$, we took into account both the radial drift caused by the interaction with the sub-Keplerian gas and the drift caused by gas accretion flow:

$\bar{v}=-\frac{2 \eta v_{\mathrm{K}} \overline{\mathrm{St}}+v_{\mathrm{g}}(1+\epsilon)}{\overline{\mathrm{St}}^{2}+(1+\epsilon)^{2}}$,

where $\eta v_{\mathrm{K}}$ is the maximum radial drift speed driven by the radial gas pressure gradient:

$\eta v_{\mathrm{K}}=-\frac{1}{2} \frac{c_{\mathrm{s}}^{2}}{v_{\mathrm{K}}} \frac{\partial \log P}{\partial \log r}$

where $v_{\mathrm{K}}$ is the Keplerian velocity of gas and $P$ is the pressure calculated taking into account contributions from nebular hydrogen and helium gas and water vapor. $\overline{\mathrm{St}}$ is the mass-weighted average Stokes number of solids at a given radial distance, which is calculated from the size distribution returned by the abovementioned algorithm. The gas accretion velocity $v_{\mathrm{g}}$ is calculated as in Eq. (3). We took into account the collective drift effect, which means that the drift velocity decreases as the solids-togas ratio increases. As most of the pebbles are settled at the midplane, we implemented the midplane solids-to-gas ratio $\epsilon=$ $\rho_{\mathrm{d}} / \rho_{\mathrm{g}}$ in Eq. (13). This equation is equivalent to the one used by Ida \& Guillot (2016) and Schoonenberg \& Ormel (2017).

\subsubsection{Fragmentation threshold}

The evolution of solids is dominated by the radial drift and by fragmentation that may stop the growth as the impact speeds increase with the Stokes number of grains (for $\mathrm{St}<1$ ). The maximum aggregate size that can be obtained before fragmentation kicks in is sensitively dependent on the fragmentation threshold velocity $v_{\mathrm{f}}$ (see Birnstiel et al. 2012):

$a_{\text {frag }} \propto \frac{v_{\mathrm{f}}^{2}}{\alpha_{\mathrm{t}} c_{\mathrm{s}}^{2}}$,

so that the choice of $v_{\mathrm{f}}$ value is in fact very important to the model outcome. $\alpha_{\mathrm{t}}$ is the midplane turbulence strength parameter that regulates impact speeds of pebbles and their settling. We purposely distinguished $\alpha_{\mathrm{t}}$ from $\alpha_{\mathrm{v}}$, the efficiency of angular momentum transport via turbulent viscosity that is used in the gas disk models. This is motivated by the fact that in many recent protoplanetary disk models the angular momentum transfer is not necessarily driven by turbulence anymore, and even if it is, a quiescent midplane layer is often formed (Dzyurkevich et al. 2013; Turner et al. 2014; Bai 2016). In most of our runs we assumed $\alpha_{\mathrm{t}}=10^{-3}$, but we discuss the impact of this value in Sect. 3.3.1.

Laboratory experiments estimated threshold velocities of around $1 \mathrm{~m} \mathrm{~s}^{-1}$ for the onset of fragmentation of silicate dust aggregates (see, e.g., Güttler et al. 2010). It is commonly accepted that aggregates containing water ice fragment at higher velocities, as their surface energies are about ten times higher than those of silicates (Wada et al. 2011; Gundlach et al. 2011; Aumatell \& Wurm 2014; Gundlach \& Blum 2015). Thus, we set the fragmentation threshold velocity according to the aggregate composition. For dry aggregates we set $v_{\mathrm{f}, \text { in }}=1 \mathrm{~m} \mathrm{~s}^{-1}$, and for aggregates containing more than $1 \%$ of water ice, we set $v_{\mathrm{f} \text {,out }}=10 \mathrm{~m} \mathrm{~s}^{-1}$. This threshold amount of ice above which we consider our aggregates to be more sticky is arbitrary, but we verified that the exact value does not make a great difference to the results of our models as the ice-to-dust ratio drops very rapidly across the snow line.

\subsubsection{Evaporation and recondensation}

We took water ice evaporation and recondensation of water vapor into account following the treatment proposed by Ciesla \& Cuzzi (2006). We traced the transport of solid ice that is incorporated into aggregates and thus migrates through the disk much faster than the gas, and the water vapor that moves at the same speed as the gas. To follow the evolution of the water vapor surface density $\Sigma_{\text {vap }}$, we solved the following transport equation:

$\frac{\partial \Sigma_{\mathrm{vap}}}{\partial t}+\frac{1}{r} \frac{\partial}{\partial r}\left[r\left(\Sigma_{\mathrm{vap}} v_{\mathrm{g}}-D_{\mathrm{g}} \Sigma_{\mathrm{g}} \frac{\partial}{\partial r}\left(\frac{\Sigma_{\mathrm{vap}}}{\Sigma_{\mathrm{g}}}\right)\right)\right]=0$,

where $v_{\mathrm{g}}$ is the gas velocity. Equation (16) is analogous to Eq. (10), which we used to follow the transport of solids.

We assumed that all the dust grains at a given orbital distance (i.e., in a given radial bin) have the same composition (i.e., ice mass ratio), which means that water is added to and removed from the dust with constant $\mathrm{d} m / m$ during recondensation and evaporation. This is consistent with an instantaneous redistribution of the ice component because of coagulation and fragmentation, which occurs when the collisional timescale

$\tau_{\text {growth }}=\frac{a}{\dot{a}} \approx \frac{1}{Z \cdot \Omega_{\mathrm{K}}}$

(see Birnstiel et al. 2012) is shorter than the radial drift timescale

$\tau_{\mathrm{drift}}=\frac{r}{\left|v_{\mathrm{r}, \mathrm{d}}\right|}$.

In our runs, this was indeed true around the snow line, where nominal timescales are $\tau_{\text {growth }} \approx 10^{3} \mathrm{yr}$ and $\tau_{\text {drift }} \approx 10^{4} \mathrm{yr}$. When the pebble pile-up is formed around the snow line, the vertically integrated dust-to-gas ratio $Z$ increases, making the growth timescale even shorter, and the radial drift speed $v_{\mathrm{r}, \mathrm{d}}$ decreases (because of the collective drift effect), making the drift timescale even longer.

At every time-step, we calculated the equilibrium pressure, which is given by the Clausius-Clapeyron equation:

$P_{\text {eq }}=P_{\text {eq }, 0} \cdot \exp \left(-\frac{A}{T}\right)$,

where the constants $P_{\text {eq }, 0}=1.14 \times 10^{13} \mathrm{~g} \mathrm{~cm}^{-1} \mathrm{~s}^{-2}$ and $A=$ $6062 \mathrm{~K}$ are taken from Lichtenegger \& Komle (1991). We compared the value of $P_{\text {eq }}$ to the water vapor pressure

$P_{\text {vap }}=\frac{\Sigma_{\text {vap }}}{\sqrt{2 \pi} H_{\mathrm{g}}} \cdot \frac{k_{\mathrm{B}} T}{\mu_{\mathrm{H}_{2} \mathrm{O}}}$,

where $\Sigma_{\text {vap }}$ is the water vapor surface density and $H_{\mathrm{g}}=c_{\mathrm{s}} / \Omega_{\mathrm{K}}$ is the gas scale-height. 
If $P_{\text {vap }}<P_{\text {eq }}$, evaporation takes place and the surface density of ice decreases by

$\Delta \Sigma_{\text {ice }}=\min \left(\sqrt{\frac{8 \pi \mu_{\mathrm{H}_{2} \mathrm{O}}}{k_{\mathrm{B}} T}} \cdot \frac{\bar{a}^{2}}{\bar{m}} \cdot P_{\text {eq }} \cdot \Sigma_{\text {ice }} \cdot \Delta t, \Sigma_{\text {ice }}\right)$,

where $\bar{a}$ and $\bar{m}$ are the average size and mass of pebble aggregates, respectively, and $\Delta t$ is the time-step. The material removed from solid ice phase is added to the vapor reservoir.

If $P_{\text {vap }}>P_{\text {eq }}$, the water vapor condenses onto grains and the surface density of ice is increased by

$\Delta \Sigma_{\text {ice }}=\min \left(2 H_{\mathrm{g}} \cdot \frac{\mu_{\mathrm{g}}}{k_{\mathrm{B}} T} \cdot\left(P_{\mathrm{vap}}-P_{\mathrm{eq}}\right), \Sigma_{\mathrm{vap}}\right)$,

which essentially means that all the excess vapor is added to the solid phase, such that the vapor pressure drops to the equilibrium pressure. The surface density added to the ice is subsequently removed from the vapor supply.

The original work of Ciesla \& Cuzzi (2006) distinguished between populations of dust and migrators (pebbles). Evaporation was considered to occur both from dust and migrators, while condensation only occurred on dust and was therefore assumed to be instantaneous. In our model, we did not explicitly make this distinction between dust and pebbles, but we assumed a continuous size distribution in every radial cell, which around the snow line is set by coagulation-fragmentation equilibrium. However, we did not model the evaporation and condensation in every size bin, but took into account the surface-weighted average size $\bar{a}$. Condensation on grains larger than micron-sized probably take some time because they have a smaller surface area available. In reality, however, vapor mostly condenses onto the smallest grains that have the greatest surface area available (see, e.g., Stammler et al. 2017). Since the snow-line region is in the fragmentation-dominated regime (see Fig. 4), the small grains should be constantly replenished, therefore we kept the assumption of instantaneous recondensation. In practice, the same assumption might have been made for evaporation, as it is very fast (a centimeter-sized pebble crossing the snow line would lose its ice content within $\tau_{\text {evap }} \approx 1$ year) in all of the runs presented in this paper.

\subsubsection{Planetesimal formation via streaming instability}

With our one-dimensional model, we cannot resolve the streaming instability that would locally condense pebbles into dense filaments, which would then gradually collapse to form planetesimals. To include the possibility of planetesimal formation via this process, we used the same approach as in Dra̧żkowska et al. (2016). At every time-step and in every radial bin, we verified whether the midplane density of pebbles exceeded unity. With the turbulence level parameter $\alpha_{\mathrm{t}} \geq 10^{-4}$ that we used in this paper, this condition is always stronger than the critical metallicity conditions proposed for a laminar disk by Dra̧żkowska \& Dullemond (2014) and Carrera et al. (2015; recently, Carrera et al. 2017, arrived at the same conclusion).

When the criterion for planetesimal formation via streaming instability is fulfilled, namely $\rho_{\mathrm{d}}\left(\mathrm{St}>10^{-2}\right) / \rho_{\mathrm{g}}>1$, part of the surface density of pebbles is transferred onto planetesimals:

$\dot{\Sigma}_{\text {plts }}=\zeta \cdot \Sigma_{\mathrm{d}}\left(\mathrm{St}>10^{-2}\right) \cdot \Omega_{\mathrm{K}}$,

with the efficiency of $\zeta=10^{-3}$, which is motivated by numerical models presented by Simon et al. (2016) and for which
Dra̧żkowska et al. (2016) found convergence of the amount of planetesimal formed, that is, the amount of planetesimal would not change significantly for higher $\zeta$ values.

\subsection{Model assumptions}

Our algorithm is limited by several assumptions, which we list here for clarity.

- We consider one-dimensional locally isothermal disk models and focus on the evolution of their midplane, where pebbles and planetesimals reside. Thus, we only consider the radial snow line and neglect effects associated with the atmospheric snow line.

- Grain sizes are set by either the coagulation-fragmentation or the growth-drift equilibrium. We do not consider the impact of evaporation and condensation on aggregate sizes. This is equivalent with assuming that the ice added during recondensation is quickly redistributed by coagulation and fragmentation. Any aggregates that would increase their size over the maximum are immediately fragmented or removed by radial drift.

- During one time-step and at a given orbital distance, all the aggregates are either in evaporation or in condensation mode. We do not consider grain curvature effects that could switch between these effects from grain to grain.

- We treat all grains as compact spheres and neglect effects of porosity.

- We assume that vertical structure of solids is always in equilibrium between settling and turbulent mixing, which leads to the dust scale-height derived by Dubrulle et al. (1995)

$H_{\mathrm{d}}=H_{\mathrm{g}} \sqrt{\frac{\alpha_{\mathrm{t}}}{\alpha_{\mathrm{t}}+\mathrm{St}}}$,

and that the water vapor is instantly mixed up to a gas scaleheight $H_{\mathrm{g}}$, even though it is released by pebbles with $H_{\mathrm{d}}<$ $H_{\mathrm{g}}$. Recently, Krijt et al. (2016a) showed that Eq. (24) breaks at high dust-to-gas ratios, when the collisional evolution is faster than the vertical redistribution.

- We assume that minimum pebble size necessary to trigger planetesimal formation via streaming instability corresponds to $\mathrm{St}=10^{-2}$ following Bai \& Stone (2010) and Dra̧żkowska \& Dullemond (2014). More recently, Carrera et al. (2015) and Yang et al. (2017) suggested that a streaming instability is also possible for smaller grains, albeit at higher metallicity. However, we find that for our assumed turbulence strength, the smaller grains do not settle in the midplane efficiently (see Eq. (24)), so that their contribution to planetesimal formation would not be possible in our models.

- The structure of our gas disks is independent of the solids evolution. This looses validity at a high dust-togas ratio when $\Sigma_{\mathrm{d}} \approx \Sigma_{\mathrm{g}}$, which occurs when the pile-up forms in our models. Recently, Gonzalez et al. (2017) and Kanagawa et al. (2017) showed that including effects of dust back-reaction on gas disk promotes the formation and sustainability of dust pile-ups.

\section{Results}

\subsection{Traffic jam inside and pebble pile-up outside of the snow line}

The common outcome of all the runs, independently of the underlying gas disk model, is that the highest solids-to-gas ratios 


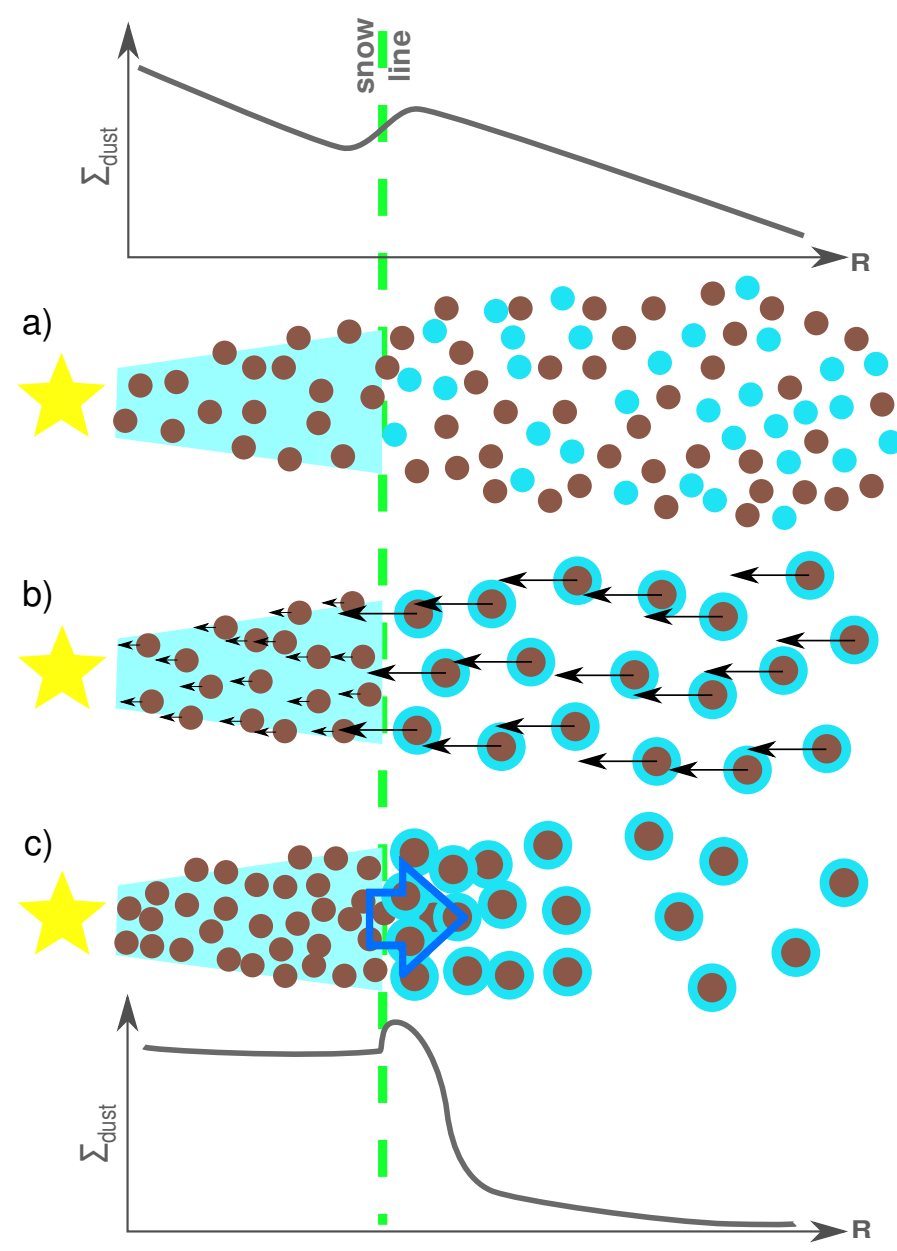

Fig. 2. Sketch explaining the processes that facilitate the formation of the snow line pile-up: $a$ ) in the initial condition, ice increases the solids density outside of the snow line; $b$ ) coagulation is more efficient for aggregates that incorporate water ice. Thus, solids grow to larger sizes and drift faster outside of the snow line. The quick drift results in an efficient delivery of the embedded refractory material, which does not drift rapidly, causing a "traffic jam" and increasing the dust concentration in the inner disk; $c$ ) the outward diffusion and recondensation of water vapor locally enhances the abundance of solids just outside of the snow line, contributing to the pile-up of icy pebbles.

are obtained in the region directly outside of the snow line. A general pattern for the formation of this snow line pile-up is shown in Fig. 2.

The dust-to-gas ratio in the inner parts of the disk is enhanced because of the general pattern of dust transport by radial drift that shifts mass inward. As explained by Birnstiel et al. (2012), if the maximum size of dust grains is regulated by fragmentation, the surface density of solids becomes proportional to $r^{-1.5}$. As the gas surface density is shallower, this redistribution leads to a depletion of the outer disk and to an increase of the solids-togas ratio in the inner disk. As demonstrated by Dra̧żkowska et al. (2016), the magnitude of this increase may already be sufficient to trigger planetesimal formation at the inner edge of the disk at a timescale of $\sim 10^{5} \mathrm{yr}$. However, this picture is complicated here by the difference in fragmentation speeds of aggregates outside and inside of the snow line.

Refractory aggregates fragment at lower impact velocities and thus reach sizes that are two orders of magnitude smaller than the icy aggregates (see Eq. (15) and Fig. 4). As the drift velocity decreases with decreasing Stokes number, dust is retained in the inner disk, causing a "traffic jam" effect. The dry dust aggregates inside of the snow line are small and well coupled to the gas, so that they undergo significant diffusion and do not form any local pile-up, unlike the icy pebbles outside of the snow line. The outward diffusion of water vapor and the subsequent recondensation causes a further increase in surface density of the icy pebbles just outside of the snow line. These pebbles are large enough to trigger streaming instability and form planetesimals. The exact location, extent, and mass of the resulting planetesimal annulus depends on the applied disk model, as discussed in Sect. 3.3.

\subsection{Fiducial simulation}

Figure 3 shows the evolution of the gas and dust surface density, including ice and water vapor as well as the forming planetesimals in the irradiated disk model with an initial dust-to-gas ratio of $Z=0.03$, which we refer to as the fiducial simulation.

The uppermost panel of Fig. 3 shows that the solid ice increases the initial surface density in the outer part of the disk. The predominant effect that shapes the evolution of solids is their redistribution, which is driven by growth and radial drift. This shifts mass inward, causing depletion of the outer parts and increase in the solids-to-gas ratio in the inner parts of the disk. Initially, the evolution outside of the snow line is dominated by fragmentation of the icy aggregates, and thus the dust surface density evolves toward $\Sigma_{\mathrm{d}} \propto r^{-1.5}$, as discussed in the previous section. After $10^{5} \mathrm{yr}$ of evolution, as the outer disk becomes depleted, it becomes dominated by the radial drift, and the surface density profile at the outer edge becomes more shallow again as it evolves to $\Sigma_{\mathrm{d}} \propto r^{-0.75}$ (Birnstiel et al. 2012).

This picture is complicated here by ice evaporation and recondensation and by the different sticking properties of wet and dry aggregates. When the grains inside of the snow line are kept small, their removal is slowed down and the high dust-to-gas ratio in the inner disk is retained. The surface density of dust inside of the snow line maintains the same profile as the gas surface density for most of the time because the aggregates are so small that their evolution is dominated by gas viscosity rather than radial drift (see Fig. 4). Ice evaporation leads to a jump in the surface density at the snow line. Recondensation of water vapor additionally increases the pebbles-to-gas ratio just outside of the snow line. The combined action of the traffic jam inside and pile-up outside of the snow line, which spreads outward through the collective drift effect (as the drift velocity decreases with increasing solids-to-gas ratio, see Eq. (13)) leads to the conditions allowing for planetesimal formation via streaming instability.

The planetesimals start to appear after $2 \times 10^{5} \mathrm{yr}$ of evolution, and their formation lasts for another $\sim 2 \times 10^{5} \mathrm{yr}$. During this time, the snow line, which marks the inner edge of the planetesimal formation region, moves inward as the disk cools down. At the same time, the planetesimal formation region slightly spreads outward because of the collective drift effect. About $20 M_{\oplus}$ of planetesimals are produced in this model. After $4 \times 10^{5} \mathrm{yr}$, the inward flux of pebbles is no longer sufficient to supply the pile-up, and the surface density of solids quickly drops, terminating the planetesimal formation phase.

\subsubsection{Pebble sizes}

Figure 4 shows pebble sizes obtained from our simplified growth and fragmentation treatment after $2 \times 10^{5} \mathrm{yr}$ of evolution (during 

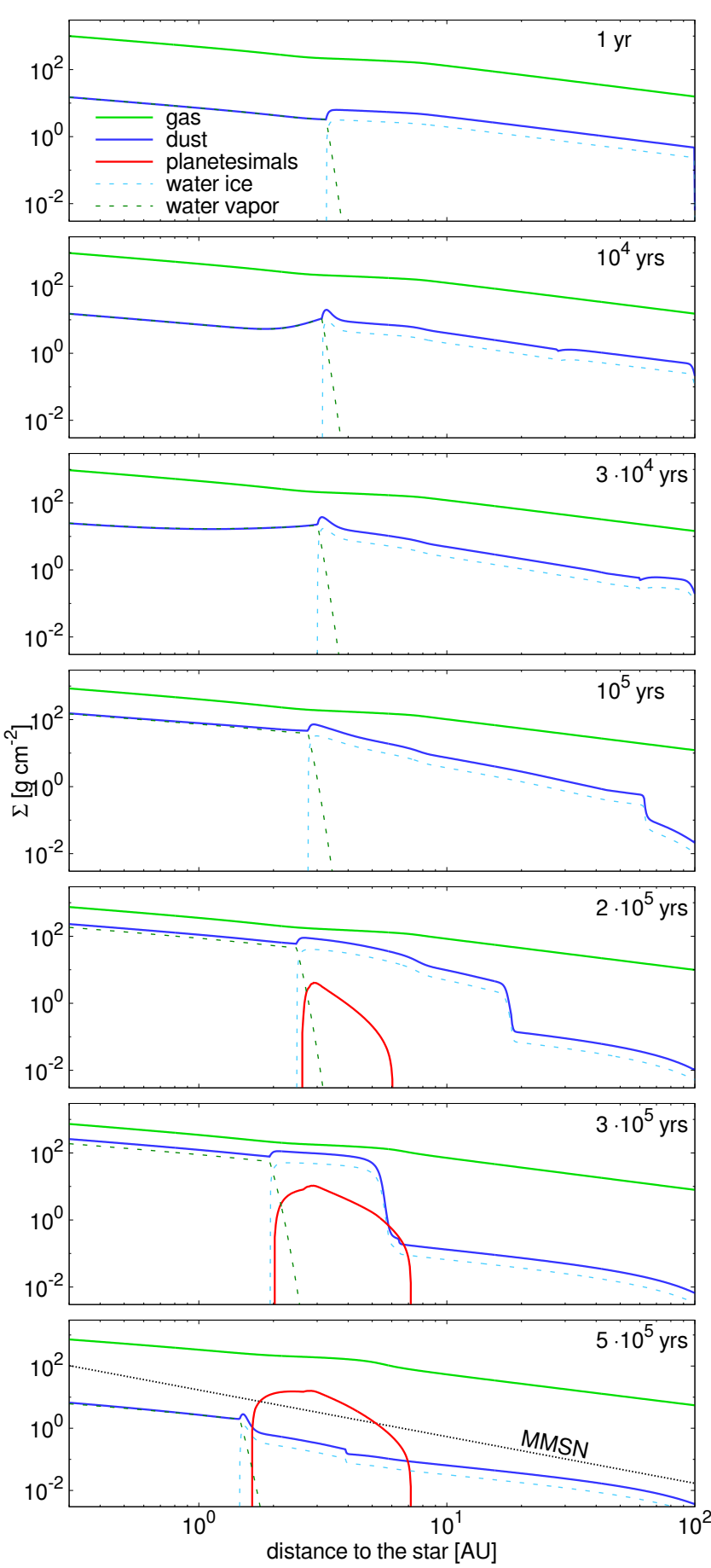

Fig. 3. Time evolution of the surface density of gas, solids, planetesimals, water ice, and water vapor in the irradiated disk model with an initial dust-to-gas ratio of $Z=0.03$. In the bottom panel, the surface density of solids in the classical minimum-mass solar nebula model (MMSN) of Weidenschilling (1977) is displayed for reference. A corresponding animation is available online.

the period of planetesimal formation) in our fiducial simulation. These effective sizes are very similar in other runs.

The dust growth follows the pattern described by Birnstiel et al. (2012) and Dra̧żkowska et al. (2016), with the inner disk being dominated by fragmentation driven by turbulence and the outer disk being gradually depleted by the radial drift before the particles have time to grow to the fragmentation limit. The modification of the fragmentation velocity described in Sect. 2.2.1 introduces the rapid change in pebble size around

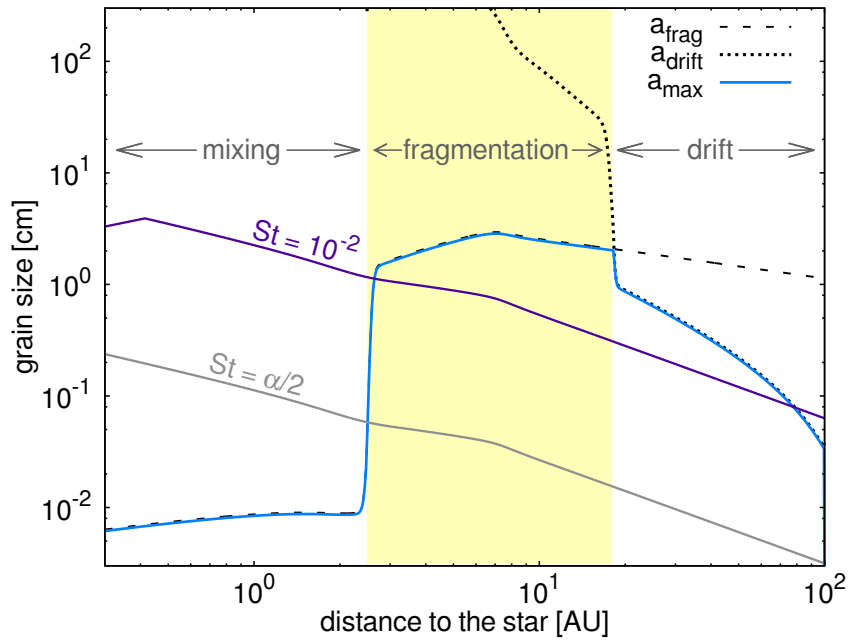

Fig. 4. Maximum pebble size $\left(a_{\max }\right)$ as a function of the radial distance after $2 \times 10^{5} \mathrm{yr}$ of evolution obtained in our fiducial run. In the outer part of the disk, this size is limited by the radial drift $\left(a_{\text {drift }}<a_{\text {frag }}\right)$, while in the inner part of the disk, it is limited by fragmentation. Aggregates that are large enough to participate in planetesimal formation via streaming instability ( $\mathrm{St}>10^{-2}$, purple solid line) are only present outside of the snow line. Inside of the snow line, the aggregate size corresponds to $\mathrm{St}<\alpha_{\mathrm{v}} / 2$ (gray solid line), which means that their transport is dominated by viscosity rather than radial drift.

the snow line. The icy pebbles outside of the snow line grow to sizes of several centimeters, corresponding to Stokes numbers of St $>10^{-2}$, which allows for planetesimal formation. The dry aggregates inside of the snow line only grow to submillimeter sizes, corresponding to $\mathrm{St}<\alpha_{\mathrm{v}} / 2$, which means that they are well coupled to the gas and follow its viscous evolution, or are in the so-called mixing regime, as discussed by Birnstiel et al. (2012). Thus, these small grains drift at much lower speed than the icy pebbles, which contributes to enhance the dust-to-gas ratio inside of the snow line and to retain icy pebble pile-up outside of the snow line through the outward diffusion of small grains.

\subsubsection{Which is more important: traffic jam or recondensation?}

The obvious question arises as to which of the two processes facilitating planetesimal formation at the snow line is more important. To answer this, we used additional models with identical setups as in the fiducial run, but with one of the mechanisms switched off. Figure 5 compares the solids-to-gas ratio around the snow line obtained in our fiducial run, the same run without recondensation of water vapor (but still including ice evaporation), and an analogous run in which the fragmentation velocity for both wet and dry aggregates was equal to $v_{\mathrm{f}}=10 \mathrm{~m} \mathrm{~s}^{-1}$, designed to exclude the traffic-jam effect inside of the snow line.

Figure 5 shows that the difference between the sticking properties of wet and dry aggregates is the dominant process facilitating formation of the solids-to-gas ratio enhancement at the snow line at every evolutionary stage of the disk. Switching the recondensation off decreases the amplitude of the solids-to-gas ratio enhancement at the snow line only by $\sim 20 \%$. On the other hand, letting the dry aggregates grow to the same sizes as the icy pebbles leads to a much more dramatic decrease in the solids-to-gas ratio in the bump. When the dry aggregates grow to pebble sizes, the solids-to-gas ratio falls more rapidly across the snow line and remains at lower levels inside of it. The surface density outside 


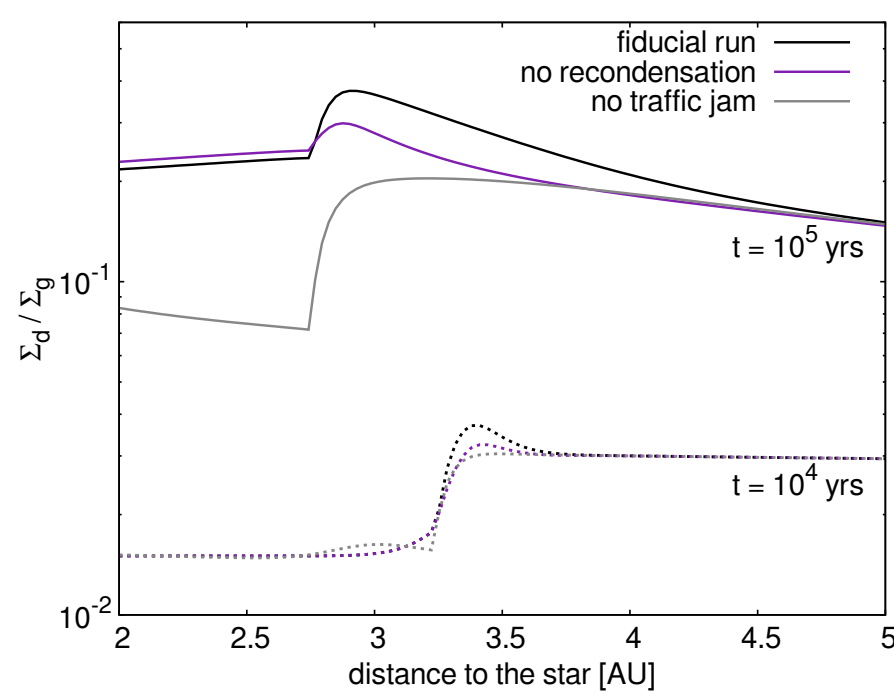

Fig. 5. Vertically integrated solids-to-gas ratio around the snow line after $t=10^{4} \mathrm{yr}$ (dotted lines) and $t=10^{5} \mathrm{yr}$ (solid lines) of evolution for our fiducial run (black lines) and for the analogous runs without the water recondensation (purple lines) and without the difference between sticking properties of wet and dry aggregates (gray lines).

of the snow line reaches lower values and the pile-up vanishes more quickly in this case.

\subsection{Planetesimal formation}

Planetesimal formation is triggered in the pile-up arising outside of the snow line in many of the runs that we performed. Table 1 summarizes the information about mass and extent of the planetesimal annulus formed in models with different underlying gas parameters, initial dust-to-gas ratio, intrinsic turbulence level, and disk extent. We note that it is significantly easier to trigger planetesimal formation when the snow line is closer to the central star. The initial metallicity of $Z=0.01$ is sufficient for planetesimal formation in the non-irradiated disk model, which is the coldest (see the middle panel of Fig. 1), while the powerlaw and the irradiated disks need at least $Z=0.02$. The reason is that close-in pile-up formation is aided by the process described by Drążkowska et al. (2016): the surface density of solids in the fragmentation-dominated regime naturally evolves to $\Sigma_{\mathrm{d}} \propto r^{-3 / 2}$, a profile steeper than the gas disk, which leads to an additional enhancement of the dust-to-gas ratio in the inner disk. The farther away the border between fragmentation-dominated and drift-dominated regions (see Fig. 4) is, the stronger the enhancement we obtain. The maximum enhancement formed by this process would fall at the inner edge of the disk (although it is diffused in our models because the small aggregates inside of the snow line fall into the mixing regime). For this reason, annuli formed closer to the central star tend to be more massive.

The inner edge of the planetesimal formation zone primarily depends on the underlying disk model and changes only slightly with the assumed metallicity. The reason is that the higher abundance of solids translates into a higher flux of pebbles that arrive at the snow line, which deliver more water vapor and thus increase the vapor pressure that determines the snow line location (see Sect. 2.2.2). At the same time, the higher the initial metallicity, the wider the planetesimal annulus. The broadening of planetesimal annulus with higher incoming pebble flux is caused by the collective drift effect. The radial drift slows down near the
Table 1. Details of the planetesimal annuli: their final mass $M_{\text {plts }}$ and radial extent $\left(R_{\text {in }}\right.$ and $\left.R_{\text {out }}\right)$, formed in runs with different underlying protoplanetary disk models, global solids-to-gas ratio $Z$, turbulence strength $\alpha_{\mathrm{t}}$, and initial disk size $R_{\text {disk }}$.

\begin{tabular}{|c|c|c|c|c|c|c|}
\hline $\operatorname{Disk}^{a}$ & $Z$ & $\alpha_{\mathrm{t}}$ & $R_{\text {disk }}^{b}$ & $M_{\text {plts }}{ }^{c}$ & $R_{\text {in }}^{b}$ & $R_{\text {out }}^{b}$ \\
\hline \multirow{5}{*}{ P-L } & 0.01 & $10^{-3}$ & 100 & - & - & - \\
\hline & 0.02 & $10^{-3}$ & 100 & 3.54 & 1.98 & 2.34 \\
\hline & 0.03 & $10^{-3}$ & 100 & 24.28 & 1.93 & 2.99 \\
\hline & 0.04 & $10^{-3}$ & 100 & 62.76 & 1.89 & 3.90 \\
\hline & 0.05 & $10^{-3}$ & 100 & 115.10 & 1.87 & 4.90 \\
\hline \multirow{4}{*}{ P-L } & 0.03 & $10^{-4}$ & 100 & 217.13 & 1.66 & 5.19 \\
\hline & 0.03 & $3 \times 10^{-4}$ & 100 & 128.79 & 1.81 & 3.44 \\
\hline & 0.03 & $3 \times 10^{-3}$ & 100 & - & - & - \\
\hline & 0.03 & $10^{-2}$ & 100 & - & - & - \\
\hline \multirow{4}{*}{ P-L } & 0.03 & $10^{-3}$ & 60 & 8.90 & 1.89 & 2.45 \\
\hline & 0.03 & $10^{-3}$ & 80 & 16.73 & 1.91 & 2.75 \\
\hline & 0.03 & $10^{-3}$ & 140 & 38.52 & 1.94 & 3.50 \\
\hline & 0.03 & $10^{-3}$ & 200 & 49.50 & 1.98 & 4.10 \\
\hline \multirow{5}{*}{ N-IRR } & 0.01 & $10^{-3}$ & 100 & 119.80 & 1.03 & 2.20 \\
\hline & 0.02 & $10^{-3}$ & 100 & 371.37 & 0.98 & 4.08 \\
\hline & 0.03 & $10^{-3}$ & 100 & 663.18 & 0.96 & 5.50 \\
\hline & 0.04 & $10^{-3}$ & 100 & 928.35 & 0.96 & 6.83 \\
\hline & 0.05 & $10^{-3}$ & 100 & 1130.9 & 0.95 & 8.00 \\
\hline \multirow{5}{*}{ IRR } & 0.01 & $10^{-3}$ & 100 & - & - & - \\
\hline & 0.02 & $10^{-3}$ & 100 & 3.32 & 2.12 & 4.33 \\
\hline & 0.03 & $10^{-3}$ & 100 & 22.50 & 1.64 & 7.16 \\
\hline & 0.04 & $10^{-3}$ & 100 & 63.31 & 1.25 & 10.37 \\
\hline & 0.05 & $10^{-3}$ & 100 & 129.93 & 1.06 & 16.99 \\
\hline
\end{tabular}

Notes. ${ }^{(a)}$ P-L: power law, N-IRR: non-irradiated, IRR: irradiated; ${ }^{(b)}$ in $\mathrm{AU} ;{ }^{(c)}$ in Earth masses.

peak of the solids-to-gas ratio, and the more pebbles are delivered to this region, the wider this peak becomes.

We stress that the collective drift effect is a critical component of our model without which it is nearly impossible to obtain significant pile-up and planetesimal formation. Recently, Schoonenberg \& Ormel (2017) arrived at the same conclusion.

\subsubsection{Impact of the turbulence strength}

All the models presented in this paper up to this point were performed assuming that the fragmentation and settling of solids is regulated by turbulence with $\alpha_{\mathrm{t}}=10^{-3}$. However, this value is rather vague, as it is very challenging to estimate the turbulence strength from observational data. Recent estimates range from $\alpha_{\mathrm{t}}<10^{-3}$ for the outer parts of the disk around HD 163296 (Flaherty et al. 2015) to $\alpha_{\mathrm{t}} \approx 10^{-2}$ in the outer parts of the TW Hya disk (Teague et al. 2016).

To test the effect of turbulence strength, we performed a suite of models in which we varied the $\alpha_{\mathrm{t}}$ parameter value and kept all the other parameters constant. For this purpose, we used the setup with a static power-law disk and an initial dust-to-gas ratio of $Z=0.03$ and varied the $\alpha_{\mathrm{t}}$ between $10^{-4}$ and $10^{-2}$. The upper panel of Fig. 6 shows that lower values of the $\alpha_{\mathrm{t}}$ parameters facilitate planetesimal formation. The lower the turbulence strength, the wider and more massive the resulting planetesimal annulus becomes. We find that no planetesimal formation is possible for $\alpha_{\mathrm{t}}$ significantly higher than our fiducial $10^{-3}$ (see Table 1) because the higher $\alpha_{\mathrm{t}}$ value reduces the size of pebbles that can 


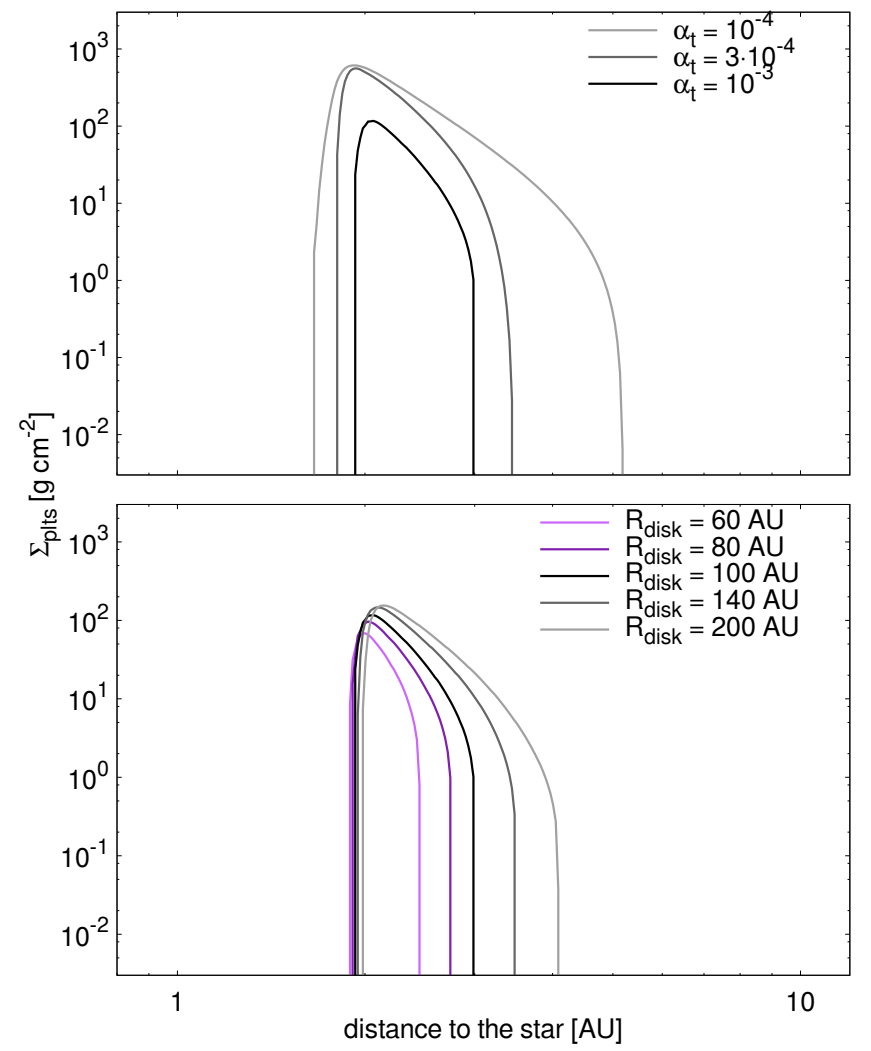

Fig. 6. Planetesimal annuli obtained in the power-law disk models with different turbulence strength parameter $\alpha_{\mathrm{t}}$ (upper panel) and initial disk extent (bottom panel). The black solid line corresponds to the same model in both panels.

grow (see Eq. (15)) and decreases the possibility of their settling (see Eq. (24)). These two factors counteract the possibility of obtaining conditions necessary to trigger the streaming instability, namely the minimum size of pebbles corresponding to $\mathrm{St}=10^{-2}$ and the midplane pebbles-to-gas ratio exceeding unity (see Sect. 2.2.3).

\subsubsection{Effect of the initial disk size}

The initial size of the protoplanetary disk is rather uncertain. Observational constraints place the outer edge of the disk anywhere between $60 \mathrm{AU}$ and several hundred AU (Andrews et al. 2009, 2010). We therefore decided to test the effect that initial disk extent has on the planetesimal formation.

The bottom panel of Fig. 6 shows the effect of the initial distribution of material. We again used the power-law disk model with an initial dust-to-gas ratio of $Z=0.03$ and turbulence strength of $\alpha_{\mathrm{t}}=10^{-3}$ and tested the change in results when the extent of this disk differed from the fiducial 100 AU (but we kept the total mass of the disk constant). The variation in the resulting planetesimal formation is not quite as pronounced as when the turbulence strength is varied, but the larger the disk, the more massive and more extended the planetesimals annulus because larger disks provide a long-lasting supply of pebbles as their growth takes longer at larger orbital distances. In other words, increasing the disk size (while keeping its mass unchanged) shifts more solid mass to its outer regions, and this reservoir can be then used to form more planetesimals.

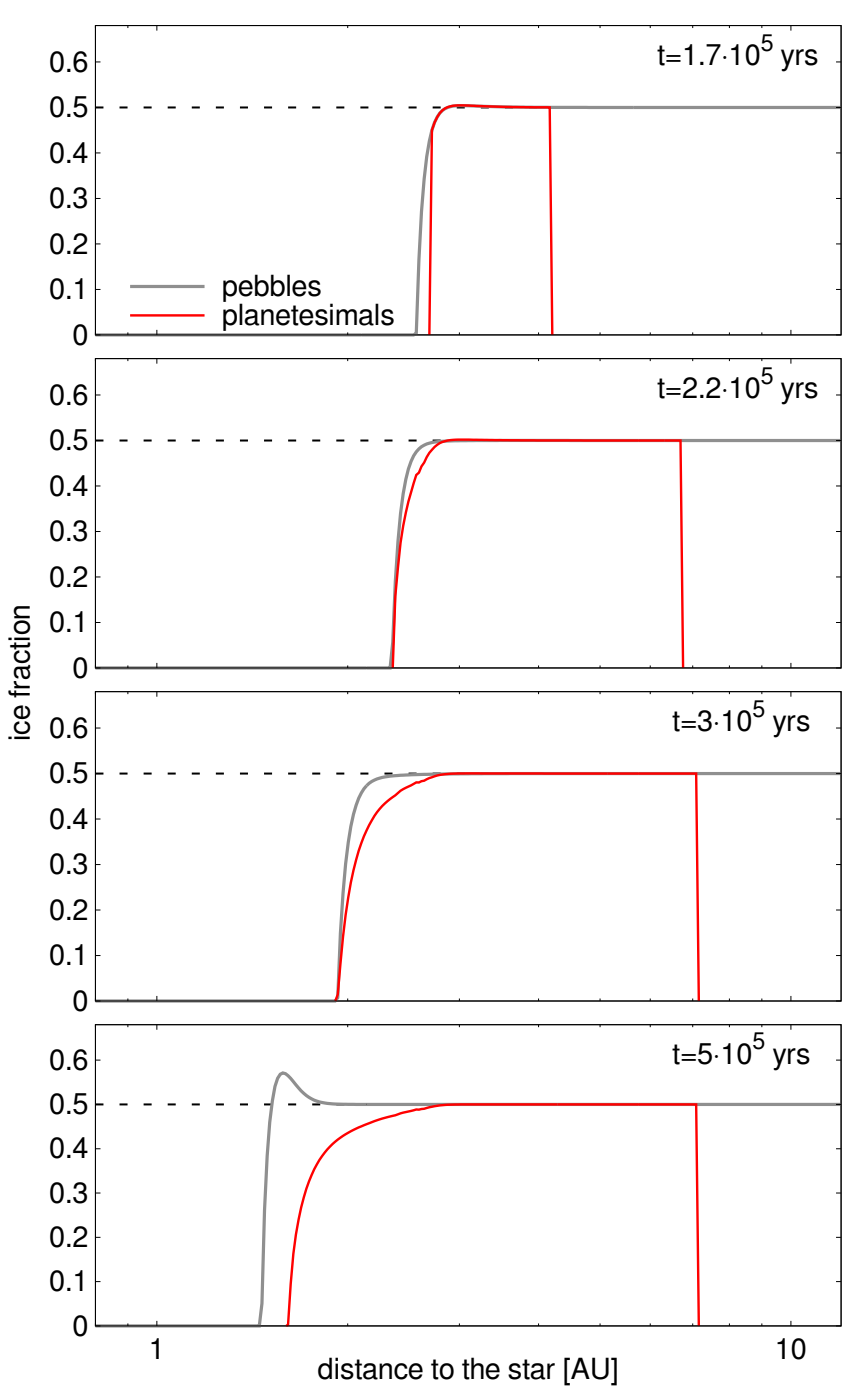

Fig. 7. Time evolution of the ice fraction of planetesimals (red) and pebbles (gray) for the irradiated disk model with an initial solids-to-gas ratio of 0.03 . The initial ice mass fraction (outside of the snow line) of 0.5 is marked with the dashed line.

\subsubsection{Pebble and planetesimal composition}

Figure 7 presents the time evolution of the ice fraction of pebbles (gray line) and ice fraction of the resulting planetesimals (red line) for the irradiated disk model with an initial metallicity of 0.03 (the same model as presented in Figs. 3 and 4). The first panel is plotted at the beginning of the planetesimal formation stage, and the last panel corresponds to a time shortly after planetesimal formation is terminated. We assumed that the composition of planetesimals reflects the composition of the pebbles from which they are forming. We started all our models with the dust outside of the snow line consisting of $50 \%$ water ice (dashed line). Evaporation removes the solid ice that is delivered to the inner disk by radial drift and turns it into vapor, part of which is able to diffuse outward and recondense, moderately enhancing the ice content of dust aggregates just outside of the snow line. As explained in Sect. 3.2.2, the main cause for the pile-up of pebbles outside of the snow line is the traffic jam arising in the inner disk and not recondensation, which is reflected here in the low amplitude of the ice fraction enhancement. When planetesimal formation starts after about $1.7 \times 10^{5} \mathrm{yr}$ of evolution, the enhancement in pebbles ice fraction completely ceases because 
the streaming instability turns the icy pebbles from outside of the snow line into planetesimals and thus hinders delivery of water to the evaporation region. It also decreases the recondensation rate. During the planetesimal formation phase, the snow line moves inward as the disk evolves, and thus the ice content of the final planetesimal population decreases more smoothly than those of pebbles, which evaporate rather quickly. After the planetesimal formation is completed $\left(\sim 4 \times 10^{5} \mathrm{yr}\right.$ of evolution), the pebble composition is set again by the interplay of evaporation and recondensation, causing the mild enhancement in ice fraction outside of the evaporation region.

\section{Discussion}

\subsection{Comparison to published work}

Armitage et al. (2016) presented a similar idea, where pebbles drift radially and pile up to reach the conditions required for planetesimal formation. However, the authors neglected dust growth and fragmentation, assuming a constant pebble size throughout the disk. In this setup, the Stokes number increases with radial distance (because the gas surface density drops, see Eq. (11)), making it easier to trigger planetesimal formation farther away from the star. At the same time, the particles farther away in the disk drift faster (because the radial drift speed depends on the Stokes number, not size, see Eq. (13)), which leads to a pile-up that is harder to generate when particle sizes are decided by fragmentation and radial drift. As derived by Birnstiel et al. (2012), in the fragmentation-dominated regime, the steady-state dust surface density is proportional to $r^{-1.5}$ and in the radial drift regime, it is proportional to $r^{-0.75}$. An analogous derivation assuming a constant dust size gives $\Sigma \propto r^{-2}$, which facilitates obtaining a high dust-to-gas ratio in the inner disk more than in our models.

Recently, Schoonenberg \& Ormel (2017) performed local models focusing on the snow line region. They took the inflow of icy pebbles into account that would be formed in the outer disk and are brought to the snow line region by radial drift and the outflow of water vapor carried with gas accretion. They found that water diffusion and recondensation enhances the surface density of icy pebbles by a factor of 3-5 outside the snow line, which is further increased if the evaporating pebbles release many small refractory "seeds" that help the pile-up through the traffic jam effect and outward diffusion. We find very similar results, both qualitatively and quantiatively, with the models presented in this paper, although they are fundamentally different by construction, as Schoonenberg \& Ormel (2017) focused on a local box and used particle approach, while in this paper we used global disk models and applied fluid approach to dust dynamics. This is very encouraging and proves that a pile-up of pebbles outside the snow line is a robust mechanism that can trigger formation of the first icy planetesimals.

On the other hand, Ida \& Guillot (2016) suggested a different scenario of planetesimal formation, namely pile-up of dry dust grains released by the icy pebbles inside of the snow line. The authors find that for a sufficiently high flux of pebbles, the dust-togas ratio increases to a point when direct gravitational instability is possible. We also find an increase in the solids-to-gas ratio inside of the snow line, but it is not as significant. The main difference is that we assumed that the small grains released from the icy pebbles quickly coagulate until they reach the fragmentation limit at about millimeter sizes and that they are vertically mixed by the turbulence, while Ida \& Guillot (2016) considered that the grains remain micron sized and their scale-height is equal to the scale-height of the icy pebbles that released them.

Both Ida \& Guillot (2016) and Schoonenberg \& Ormel (2017) stressed the importance of a sufficiently high pebble mass flux for the possibility of planetesimal formation. In their models, the pebble flux was a free parameter since they did not self-consistently include dust growth to pebble sizes and their drift. With our models, we can measure the pebble flux incoming to the snow line region. For the power-law disk model, which is similar to the models used in the quoted papers, we measured a ratio of pebble mass flux to gas mass flux during the planetesimal formation stage on the order of 0.5 , which Schoonenberg \& Ormel (2017) also found sufficient for triggering planetesimal formation via streaming instability outside of the snow line.

\subsection{Implications for planet formation}

Our results suggest that the formation of planetesimals is simpler in the direct vicinity of the water evaporation front. This could naturally explain the fast formation of Jupiter in the solar system (Kruijer et al. 2017). The surface density obtained in the planetesimal annulus in all of our runs is higher than predicted by the minimum-mass solar nebula models (Weidenschilling 1977; Hayashi 1981). Starting from a high mass concentration translates into a faster growth of planetesimals into planetary embryos and the planetary cores. It is known that an enhancement of about ten times over the solids surface density corresponding to the minimum-mass solar nebula is necessary to allow for Jupiter core formation before the gas disk dispersal (Pollack et al. 1996; Ikoma et al. 2000; Kobayashi et al. 2010). The fast formation of a gas giant outside of the snow line could possibly halt the delivery of water to the inner part of planetary system (Morbidelli et al. 2015). If such a barrier is not formed quickly, it may be problematic to explain the low water content of terrestrial planets in the solar system (Sato et al. 2016).

Our results, including the self-consistent surface densities and composition of planetesimals and pebbles, may be used as an input to models that study the later stages of planet accretion, particularly those that discuss the pebble accretion process, when the planetary cores grow by accreting not only planetesimals, but also the leftover pebbles that were not incorporated during the planetesimal formation stage. The radial dependence of sizes and radial flux of pebbles that we can extract from our results are important parameters of pebble accretion models (Ormel \& Klahr 2010; Lambrechts \& Johansen 2014; Levison et al. 2015; Visser \& Ormel 2016).

One prominent consequence of the planetesimal formation mechanism we discussed is that the first planetesimals are water rich (see Fig. 7). As a consequence, planetary cores formed from these planetesimals would also be water rich. For lowmass planets (without a massive gas envelope), large amounts of water may be detrimental for habitability (see Alibert et al. 2013; Kitzmann et al. 2015; see, however, Levi et al. 2017, for another view). The planetesimal formation mechanism we described here could therefore imply that the majority of low-mass planets are not habitable. However, recent models show that the low-mass short-period planets detected by the Kepler mission are probably water-poor (see, e.g. Jin \& Mordasini 2017). In the framework of our planetesimal formation model, this implies that other mechanisms, facilitating efficient water loss from existing planetesimals or allowing the formation of dry planetesimals, are at work to prevent the accumulation of water on these short-period planets. 


\section{Summary}

We addressed the connection between dust evolution and planetesimal formation, which is still one of the least certain aspects of planet formation theory. As dust growth is hindered by collisional fragmentation and radial drift, continuous growth from micron to planetesimal sizes appears to be improbable. We propose that the first planetesimals form via streaming instability, in a pile-up of icy pebbles generated outside of the snow line.

The water snow line is a favorable location for planetesimal formation as large icy pebbles efficiently deliver water and embedded refractory material to the inner part of the disk. The water vapor is partially mixed outward by diffusion and recondenses just outside of the snow line, locally enhancing the solids-togas ratio. At the same time, the less sticky dry aggregates inside of the snow line drift at much lower speed, creating the traffic-jam effect and helping to reach the high dust concentration that is needed to form planetesimals in the streaming instability scenario.

A relatively compact annulus of icy planetesimal is a common result of our simulations, performed with three diverse protoplanetary disk models and different input parameters. The main condition we find for making this outcome feasible is that the turbulence strength cannot be too high: the corresponding $\alpha_{\mathrm{t}}$ parameter must remain equal to or below $10^{-3}$. Moreover, the farther away the snow line is located, the higher the disk metallicity that is needed to allow for planetesimal formation.

On a more general level, this work as well as similar studies (Dra̧żkowska et al. 2016; Carrera et al. 2017; Gonzalez et al. 2017) indicate that the dust distribution during and after the planetesimal formation stage is very different from the commonly assumed power laws, as a significant redistribution of solids must take place before the conditions necessary for planetesimal formation are met. In particular, the solids-to-gas ratio is significantly increased at the location where planetesimals form, which should facilitate a faster accretion of the final planets.

Acknowledgements. We thank the anonymous referee for their detailed reports that helped us to improve this paper. This work has been carried out within the framework of the National Centre for Competence in Research PlanetS supported by the Swiss National Science Foundation. The authors acknowledge the financial support of the SNSF.

\section{References}

Alibert, Y., Mordasini, C., Benz, W., \& Winisdoerffer, C. 2005, A\&A, 434, 343 Alibert, Y., Carron, F., Fortier, A., et al. 2013, A\&A, 558, A109

Andrews, S. M., Wilner, D. J., Hughes, A. M., Qi, C., \& Dullemond, C. P. 2009, ApJ, 700, 1502

Andrews, S. M., Wilner, D. J., Hughes, A. M., Qi, C., \& Dullemond, C. P. 2010, ApJ, 723, 1241

Armitage, P. J., Eisner, J. A., \& Simon, J. B. 2016, ApJ, 828, L2

Aumatell, G., \& Wurm, G. 2014, MNRAS, 437, 690

Bai, X.-N. 2016, ApJ, 821, 80

Bai, X.-N., \& Stone, J. M. 2010, ApJ, 722, 1437

Banzatti, A., Pinilla, P., Ricci, L., et al. 2015, ApJ, 815, L15

Birnstiel, T., Dullemond, C. P., \& Brauer, F. 2010, A\&A, 513, A79

Birnstiel, T., Klahr, H., \& Ercolano, B. 2012, A\&A, 539, A148

Bitsch, B., Johansen, A., Lambrechts, M., \& Morbidelli, A. 2015, A\&A, 575, A28
Brauer, F., Henning, T., \& Dullemond, C. P. 2008, A\&A, 487, L1 Carrera, D., Johansen, A., \& Davies, M. B. 2015, A\&A, 579, A43 Carrera, D., Gorti, U., Johansen, A., \& Davies, M. B. 2017, ApJ, 839, 16 Ciesla, F. J., \& Cuzzi, J. N. 2006, Icarus, 181, 178

Cridland, A. J., Pudritz, R. E., \& Birnstiel, T. 2017, MNRAS, 465, 3865 Cuzzi, J. N., \& Zahnle, K. J. 2004, ApJ, 614, 490

Dra̧żkowska, J., \& Dullemond, C. P. 2014, A\&A, 572, A78

Dra̧żkowska, J., Windmark, F., \& Dullemond, C. P. 2013, A\&A, 556, A37

Drążkowska, J., Alibert, Y., \& Moore, B. 2016, A\&A, 594, A105

Dubrulle, B., Morfill, G., \& Sterzik, M. 1995, Icarus, 114, 237

Dzyurkevich, N., Turner, N. J., Henning, T., \& Kley, W. 2013, ApJ, 765, 114

Estrada, P. R., Cuzzi, J. N., \& Morgan, D. A. 2016, ApJ, 818, 200

Flaherty, K. M., Hughes, A. M., Rosenfeld, K. A., et al. 2015, ApJ, 813, 99

Gonzalez, J.-F., Laibe, G., \& Maddison, S. T. 2017, MNRAS, 467, 1984

Gundlach, B., \& Blum, J. 2015, ApJ, 798, 34

Gundlach, B., Kilias, S., Beitz, E., \& Blum, J. 2011, Icarus, 214, 717

Güttler, C., Blum, J., Zsom, A., Ormel, C. W., \& Dullemond, C. P. 2010, A\&A, 513, A56

Hartmann, L., Calvet, N., Gullbring, E., \& D'Alessio, P. 1998, ApJ, 495, 385

Hayashi, C. 1981, Prog. Theor. Phys. Suppl., 70, 35

Hughes, A. L. H., \& Armitage, P. J. 2012, MNRAS, 423, 389

Ida, S., \& Guillot, T. 2016, A\&A, 596, L3

Ikoma, M., Nakazawa, K., \& Emori, H. 2000, ApJ, 537, 1013

Jin, S., \& Mordasini, C. 2017, ArXiv e-prints [arXiv: 1706.00251]

Johansen, A., Oishi, J. S., Mac Low, M.-M., et al. 2007, Nature, 448, 1022

Johansen, A., Klahr, H., \& Henning, T. 2011, A\&A, 529, A62

Kanagawa, K. D., Ueda, T., Muto, T., \& Okuzumi, S. 2017, ApJ, 844, 142

Kataoka, A., Tanaka, H., Okuzumi, S., \& Wada, K. 2013, A\&A, 557, L4

Kitzmann, D., Alibert, Y., Godolt, M., et al. 2015, MNRAS, 452, 3752

Kobayashi, H., Tanaka, H., Krivov, A. V., \& Inaba, S. 2010, Icarus, 209, 836

Kowalik, K., Hanasz, M., Wóltański, D., \& Gawryszczak, A. 2013, MNRAS, 434, 1460

Kretke, K. A., \& Lin, D. N. C. 2007, ApJ, 664, L55

Krijt, S., Ciesla, F. J., \& Bergin, E. A. 2016a, ApJ, 833, 285

Krijt, S., Ormel, C. W., Dominik, C., \& Tielens, A. G. G. M. 2016b, A\&A, 586, A20

Kruijer, T. S., Kleine, T., Burkhardt, C., \& Budde, G. 2017, in Lunar and Planetary Science Conf., 48, 1386

Lambrechts, M., \& Johansen, A. 2014, A\&A, 572, A107

Levi, A., Sasselov, D., \& Podolak, M. 2017, ApJ, 838, 24

Levison, H. F., Kretke, K. A., \& Duncan, M. J. 2015, Nature, 524, 322

Lichtenegger, H. I. M., \& Komle, N. I. 1991, Icarus, 90, 319

Morbidelli, A., Lambrechts, M., Jacobson, S., \& Bitsch, B. 2015, Icarus, 258, 418

Okuzumi, S., Tanaka, H., Kobayashi, H., \& Wada, K. 2012, ApJ, 752, 106

Ormel, C. W., \& Klahr, H. H. 2010, A\&A, 520, A43

Pollack, J. B., Hubickyj, O., Bodenheimer, P., et al. 1996, Icarus, 124, 62

Ros, K., \& Johansen, A. 2013, A\&A, 552, A137

Sato, T., Okuzumi, S., \& Ida, S. 2016, A\&A, 589, A15

Schoonenberg, D., \& Ormel, C. W. 2017, A\&A, 602, A21

Shakura, N. I., \& Sunyaev, R. A. 1973, A\&A, 24, 337

Simon, J. B., Armitage, P. J., Li, R., \& Youdin, A. N. 2016, ApJ, 822, 55

Stammler, S. M., Birnstiel, T., Panić, O., Dullemond, C. P., \& Dominik, C. 2017, A\&A, 600, A140

Stevenson, D. J., \& Lunine, J. I. 1988, Icarus, 75, 146

Teague, R., Guilloteau, S., Semenov, D., et al. 2016, A\&A, 592, A49

Turner, N. J., Fromang, S., Gammie, C., et al. 2014, Protostars and Planets VI, 411

Visser, R. G., \& Ormel, C. W. 2016, A\&A, 586, A66

Wada, K., Tanaka, H., Suyama, T., Kimura, H., \& Yamamoto, T. 2011, ApJ, 737, 36

Wang, X.-M. 2015, MNRAS, 449, 1084

Weidenschilling, S. J. 1977, Ap\&SS, 51, 153

Wuchterl, G., Guillot, T., \& Lissauer, J. J. 2000, Protostars and Planets IV, 1081

Yang, C.-C., Johansen, A., \& Carrera, D. 2017, A\&A, 606, A80

Youdin, A. N., \& Goodman, J. 2005, ApJ, 620, 459 